\title{
Study and Realization of Circuit Analysis Online Test System Based on Web
}

\author{
http://dx.doi.org/10.3991/ijoe.v10i2.3101 \\ Peijiang Chen \\ School of Automobile, Linyi University, Linyi, China
}

\begin{abstract}
Circuit analysis is an important professional basic course for the majors of mechanical engineering and electrical engineering, which has basic, practical and advanced features. In order to improve the teaching quality and to provide the services to teachers and students, the construction method of online self-test system of circuit analysis course is studied. Aiming at the characteristics of self-test system, the relative application technologies, including the development modes and development software, are analyzed. According to the performance requirements, the online test system is divided into three modules, administrator module, teacher module and student module, and the main functions are discussed in detail. The system is realized based on ASP.NET, the discussions are emphasized on the principles and strategies of intelligent paper combination, and system security mechanism. The system is convenient to provide the services of self-test and course evaluation for students, and to promote the teaching management and enhance the teaching quality.
\end{abstract}

Index Terms-Online Test; Circuit Analysis; Web; Paper generation; Network Security

\section{INTRODUCTION}

Circuit analysis is a compulsory basic course for the students of many majors, such as mechanical engineering, vehicle engineering, electrical Engineering, and so on. The course is very important and has direct impact on the following course study and practical work. Because the course has many knowledge points and strong applicability, a lot of students feel that the concepts of circuit analysis are very abstract and their logicality are strong, then, it is hard to master and the pass rate of course examination is low relatively.

With the rapid development of network information technology and computer technology, computer-aided education is being more and more widely appreciated [1]. Online test management using computer has become an important branch and part in education fields, and it is an effective method to improve the teaching quality. It can provide for many students with the environment of selftest and evaluation. It can be seen as a complementary learning way besides the normal teaching activity. Online test system can strengthen the quality education and the teaching management process, feedback the learning information to help students give themselves the position of learning outcomes.

In this study, a simple online test system based on Web by using the programming language of ASP.NET are designed and realized, which can be applied to the course of circuit analysis, and it can provide help for the teaching, learning, self-test and course evaluation.

\section{System DeVelopment Models AND TECHNOLOGIES}

\section{A. Development Models Analysis}

There are two structural models for developing information systems based on web, they are $\mathrm{C} / \mathrm{S}$ and $\mathrm{B} / \mathrm{S}$, the former is a two-layer structure model, the other is a three-layer structure model.

1) $\mathrm{C} / \mathrm{S}$ development model

$\mathrm{C} / \mathrm{S}$ is a distributed computing model. It divides application into many appropriate tasks which are carried out respectively by many machines to implement the entire application. There are a variety of interactive relationships among these tasks, in which the basic one is request-response. That is the client brings forward the processing request of information or data to the server which completes the appropriate treatment and feedback the results to the client as a response aiming at the request.

$\mathrm{C} / \mathrm{S}$ model achieves processing requirements with two ends, and it has higher performance than the file server system. The model can complete the request from the clients and realizes distributed application processing. The server manages data for several client application programs, and the client program sends requests and analyzes the data received from the server [2].

Although $\mathrm{C} / \mathrm{S}$ structure is very mature in technology, there are many disadvantages, such as poor flexibility, difficult to upgrade, more work for maintenance, poor system security, and so on, it is difficult to adapt to the current development of information and network technologies.

2) B/S development model

$\mathrm{B} / \mathrm{S}$ model is improved according to the defects of the two-layer structure. It is a new MIS system platform model based on Web technology. It divides the server of the traditional $\mathrm{C} / \mathrm{S}$ model into a data server and one or more application servers. Then, a client server network with three layers is constructed.

In recent years, Intranet, which is based on Web technology, is being widely used. The client browser with lost cost and easy usage method can access to the Web site and look up the required data. The consistency of the operation interfaces of the client overcomes the inconsistencies of website data brought by a variety of procedures in the two-layer structure $\mathrm{C} / \mathrm{S}$ mode client. The open of server and standard connectivity plan greatly enhance the connection between the website with the 
external. At the same time, the dynamic and interactive information distribution improves the service quality and increases the business opportunities.

Although B/S development model adopts the application server architecture, it resolves the shortcomings of $\mathrm{C} / \mathrm{S}$ mode better. The application system using $\mathrm{B} / \mathrm{S}$ mode is easier to use, and the users can accept and learn it easily. The system can be maintained conveniently, in addition to the operating system and browser, it done not need any other client software, and client presentation logic is downloaded from the Web server.

\section{B. Development Technologies Analysis}

There are many technologies to of develop Web applications, the most popular dynamic web development technologies includes ASP, PHP, JSP, ASP.NET, and so on.

ASP.NET is an Internet programming technology developed by Microsoft, which is the collection of ASP and .NET. It is not a simple upgrade of ASP, but a Web development platform, which can provide the necessary services of creating Web applications. ASP.NET provides a powerful server control structure, to make web developing easier, while ASP.NET also has a more concise event-based programming model which enables Web developing closer to the traditional windows Form programming. In addition, ASP.NET pages are compiled, so the applications running in the Web server greatly exceed the ASP applications on the performance and scalability. In short, ASP.NET is simple and efficient, which can visually develop complex Web applications.

\section{SYSTEM OVERALL DESIGN}

\section{A. Performance Requirements}

For online test systems, there are several key factors.

Practicability: For the development of online test system, practicability must be put first. It should meet the requirements of the general objective questions, such as single choice, true or false question, filling in the blanks, and so on [3]. After understanding a set of basic requirement definition in detail, we can quickly construct a preliminary version of the target system. According to the evaluation and usage results of a variety of users, an improved version can be got after modifying and adding. Repeating the above processes until the users satisfy, we ultimately get the complete version.

Reliability: An important aspect of service quality is to access the information within the response time. It means that we should provide the necessary information within the expect time tor the users who need Internet application or information. We can suppose that the examination system with hundreds or thousands of students accessing at the same time, once the server is blocked or collapse, it will bring unimaginable trouble. In addition, long delay will give the candidates inconvenience.

Scalability: Scalability refers that the system can ensure the sustainable growth to meet the customer needs and business complexity requirements. We identify the system as a dynamics model. It usually is small at the beginning, but it grows exponentially with the development of system demand. This growth is very rapid, not only in the number of users supported, but also in the complexity and integration of user service. For the online test system, this scalability is particularly important, because the improvement of test function is a gradual process. The original examination system needs to be improved with the requirement of student and the development of technology, then, the scalability is essential.

Security: It means that the system can protect data or basic structure to avoid malicious attack. Security can prevent risks and make system safe through providing adequate protection for the confidentiality, integrity and reliability of the information. The security is the essential factor for any system.

Manageability: Manageability refers that the system ca be managed easily. In order to ensure the normal operation and management, it involves the following factors, maintaining system service and the basic structure, tools, administrators and technical staff needed to serve normal work. The management and monitor of the system must be completed remotely.

\section{B. System Functional Modules}

According to the characteristics of the circuit analysis course, the online test system is divided into three modules which are administrator module, teacher module and student module. Administrator module mainly manages and controls the system including the functional permission of users, the setting of examination environment. Teacher module includes questions management, paper management and paper marking. Students module includes online training, online exam and score inquiry.

The function module of the online test system is shown in Fig. 1.

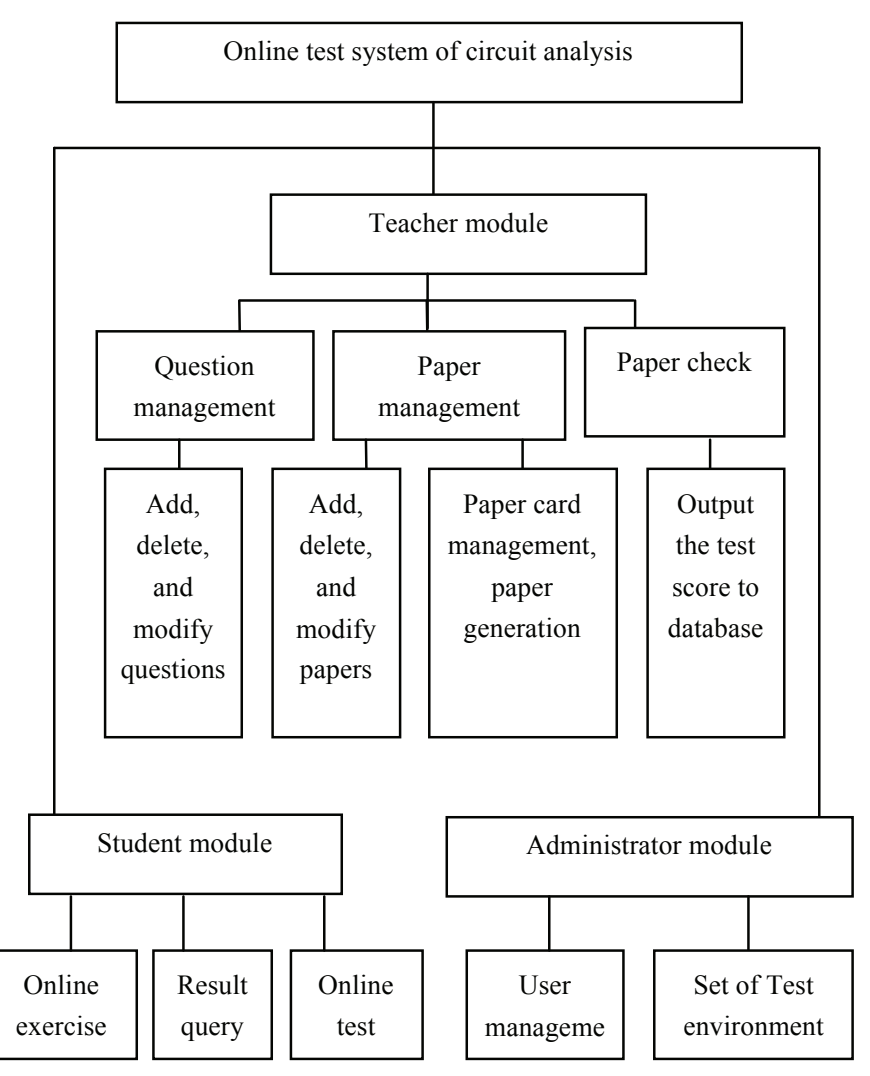

Figure 1. Function Modules of Online Test System 
The functions of main modules are as follows.

Paper management: This module is the core of the system, and the main function is to complete the random paper generation and artificial paper generation. Random paper generation means that the teacher makes paper requests from the different aspects of chapter, question number, question type, difficulty, reliability through controlling parameters input according to paper cards, and the computer automatically extracts from the question bank to generate paper by the question extraction algorithm, which can quickly organize the papers. Artificial paper refers that the user can arbitrarily select questions to generate paper using the relevant topics according to test requirements, which is more flexible. We often combine the two methods to get a paper. Firstly, we randomly produce paper, then, manually modify it. So, we can get a more reasonable paper. After producing the paper, we can browse paper effect through preview function.

Question management: This module mainly inputs, modifies, queries, adds and deletes the information of all kinds of questions. According to different types, sections and difficulty, we input questions and directly stock them in the question bank, which can be used later.

Paper marking: The module provides teachers with online checking and marking function. The result and scores of the student online test are saved in the system database. Teachers mark the subjective questions step by step according to the reference answers, and the objective questions are automatically scored by the system and after getting the results, they can be automatically or manually entered into the database.

Online exercise: The online exercise can provide exercises of each chapter of circuit analysis courses. Students can access the various sections of the test exercises, and submit after completion. The system gives answers and scores for the objective questions, and reference answers for the subjective questions.

Online test: In order to test students, teachers publish the corresponding papers to let students complete the examination within the specified time. Students enter the examination room, do questions on line, submit answers at the end of time or in the middle of test[4].

Result query: After completing the online test, teachers check papers and get scores. Students can inquiry their scores of each test.

\section{System Key TeChNOlOGIES}

\section{A. Automatic Test Paper Generation}

Principle of automatic paper generation: Automatic paper generation by computer should follow the principles.

Universality: The system should support the establishment of requirements of a variety of test types to produce normal papers.

Simplicity: It suits for the test system of distant education and routine teaching.

Random: After giving paper indicator, the system can automatically realize random combination, and also can select specific questions according to specific indicator.

Rationality: The paper question should cover wide range, and there are not associated questions simultaneously in the same paper, and the entire score and time should meet the conventional indicators.

Adaptability: When generating papers, we should adjust the knowledge point, difficulty, teaching level and other characteristics according to the actual teaching.

Strategies of Paper Generation: The strategy of test paper generation is the core of the examination system. Different test has different focus, the strategy will change correspondingly. There are many algorithms, and the common algorithms include random selection method, retrospective testing and genetic algorithm. The efficiency and quality of automatic paper generation entirely depends on the design of the question extracting algorithm. In order to meet the different needs of users, the algorithm must be random, scientific and reasonable.

The system can define the distribution of difficulty, knowledge points, question types and chapters according to the requirements of teachers. The system automatically selects questions from the bank to meet the needs of the most examinations. Genetic algorithm is process of cyclic iteration which can make the individual of group calculate continuously towards the optimal solution, until to the optimal solution [5].

The process of genetic algorithm is shown in Fig. 2.

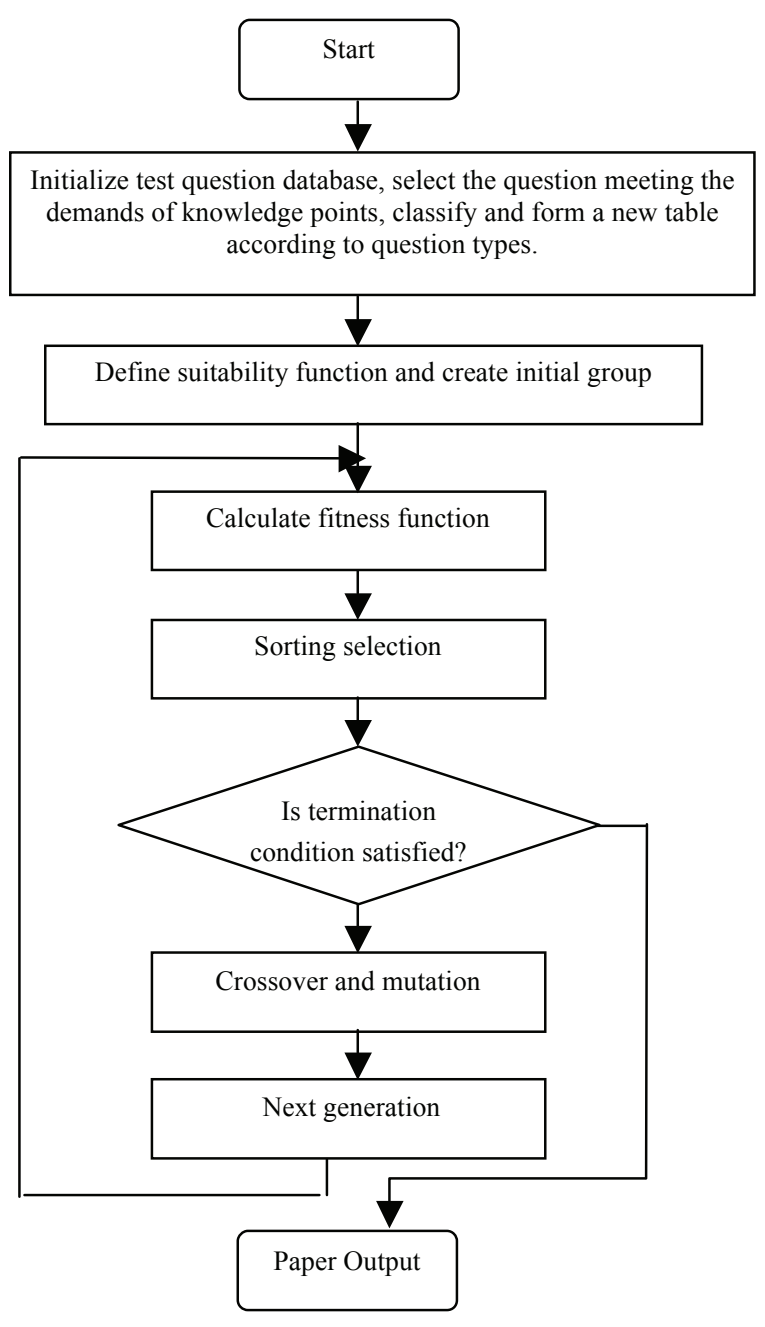

Figure 2. Process of genetic algorithm 


\section{B. Network Security}

It is very important for the data security and confidentiality because of the special requirements of online test system. Confidentiality refers that all online user information should be confidential, and the contents of different types of users should be confidential with each other. Safety refers that the each event users participate is a safe process, and the servers should track the actions of all users. In order to ensure the security and confidentiality, we must make that the students can not cheat online, monitor the test process in real-time. They can be realized by the following ways primarily.

1) All the critical data of the system are transmitted and stored by encryption technology; even super users can not modify the critical data without authorization.

2) All users who enter the test system must be authenticated, the system prohibits many persons enter with a single login account to prevent cheating.

3 ) In order to ensure the stability of the system, it sets restrictions of the maximum login number. When it exceeds the restriction, the system will prohibit the login of subsequent users. If the user has not any action after entering the system, the system will cancel all operations and the user must login it again.

4) The system assigns different operation permission to different users, supports operation according to authority to ensure the publicity and privacy of data. It prohibits illegal operation to prevent the system from intentional or unintentional destruction. It avoids users from viewing the source files and copies.

5) The administrator can allow or prohibit certain users to login the special system. The illegal exited user can login the system again only with the admission of the administrator [6].

\section{CONCLUSION}

The online test system realized in this paper, which is based on B/S model and combined with ASP.NET and SQL database, convert the conventional examination model into a novel, objective and practical test method. The test system is very practical, and has become an important component of the modern education mode. The test system is used in the course of circuit analysis, the result shows that the system can be running well on the LAN, it is operated easily and the interface is friend. The system is suitable for students to practice after class, selftest and course evaluation. The system can facilitate the online test, make the feedback of teaching information rapid, let the management and control of the teaching process easy and promote the level of the teaching management and teaching evaluation, and it is an important means to improve the teaching quality.

\section{REFERENCES}

[1] X. Tao, and H. L. Chen, "Design and Realization of Online Examination System for Circuit," Research and Exploration in Laboratory, vol. 27, no. 7, pp. 83-86, 2008.

[2] C. Hou, "Web-based Online Test System," Master Thesis, Changchun, China: Jilin University, 2009.

[3] LI Jingyun: Design and Implementation of Web-based Online Test System. Master Degree Thesis, Beijing, China: Beijing University of Posts and Telecommunication, 2009.

[4] X. Z. Li and J. Ma, "Design and Development of Test System Based on Web," Journal of Weifang Education College, vol. 20, no. 2, pp.25-26, 2007).

[5] J. Cui, "Design and Implementation of Online-Test System Based on Web," Master Thesis, Tianjin, China: Tianjin University, 2010.

[6] Z. G. Chen and J. Zhang, "The Implementation of the Online Examination," Journal of Henan University (Natural Science), vol. 33, no. 3, pp. 69-71, 2003.

\section{AUTHORS}

Peijiang Chen is with the School of Automobile, Linyi University, Linyi, Shandong, 276000, China (e-mail: chenpeijiang@163.com).

This work was supported in part by Vocational Education and Adult Education "twelfth Five-Year Plan" Subject of Shandong Province, P. R. China (No. 2011ZCJG277).

Submitted, August, 8, 2013. Published as resubmitted by the authors on March, 9, 2014. 